\title{
You Need the AI Community-and the AI Community Needs You!
}

\author{
Ute Schmid ${ }^{1}$
}

Published online: 6 June 2015

(C) Springer-Verlag Berlin Heidelberg 2015

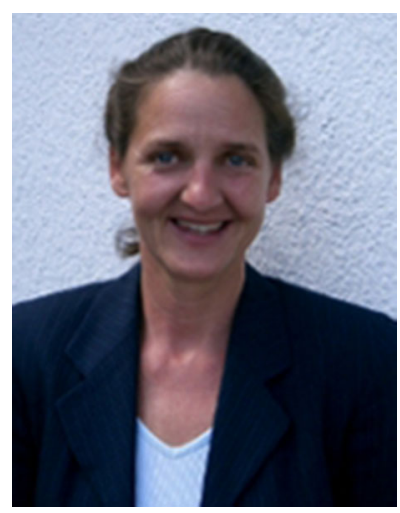

Dear readers,

How could AI research remain active and productive without the AAAI as international, the ECCAI as European, and the GI Technical Committee Künstliche Intelligenz as German representation of the common interests of all researchers in this domain? What about the important conferences, be it the AAAI conferences or the IJCAI, the ECAI, and-yes, also-the German AI Conference KI? What about the community building journals, the AI Magazine, the AI Communications, and-yes, also- the German Journal on Artificial Intelligence, KI, whose editorial for volume 29, issue 3 you are currently reading? Who would represent the interests of $\mathrm{AI}$ at national funding organizations such as the German DFG or on an European or international level? Who would care for the continuity of the main conferences?

The probability that you, the reader, are a paying member of AAAI or GI is moderately high. However, the probability that you, the reader, are also an active member of your community is relatively low. Research communities need money, but - much more importantly - they need people, people that give their time to the cause! Serving

Ute Schmid

ute.schmid@uni-bamberg.de

1 Cognitive Systems, Faculty Information Systems and Applied Computer Science, University of Bamberg, 96045 Bamberg, Germany your community does not just mean that you have a position which you can list in your CV, it means work. Definitely, it is more glamorous and you have more impact if you serve in an international society rather than in a German one and it is very important that there are researchers working in Germany who are active in international societies. Nevertheless, I strongly believe that-even if the world (at least a larger part of it) is a global villagecommunities first need to grow in the nearer surrounding where you share a common background, can meet more often and talk in person.

For this reason, I want to bring to your notice some people who are giving their time and their inspiration to the German AI community: The speaker of the German Technical Committee Künstliche Intelligenz Antonio Kröger, as well as the vice-speaker Stefan Wölfl for the time, effort and enthusiasm they give to the management and the representation of the German AI community; the chairs and organizers of the German AI Conference 2015, with the general chair Steffen Hölldobler from TU Dresden for offering us an attractive program at an attractive location for KI'15 which hopefully will result in many submissions of high quality; Frieder Stolzenburg and Marco Ragni who are the speakers of the special interest group Cognition within the GI Technical Committee "Knstliche Intelligenz" and the guest editors of the current issue for keeping the topic of cognitive oriented AI research active and visible in the German AI landscape, and the editorial board of the German Journal of Artificial Intelligence with the editor in chief KlausDieter Althoff whose member I had the pleasure to be from 2007 until now.

During my time in the editorial board I learned a lot, most importantly, how to motivate authors, guest editors, and reviewers to deliver within time. I got to know 
colleagues from other areas of AI research and thereby broadened my horizon. I gained some insight in the publishing process and had the chance to look behind the scenes of Springer-special thanks to Hermann Engesser and Dorothea Glaunsinger for all the productive meetings at Springer in Heidelberg! So-yes, it was work, and more often than not, the work had to be done when other deadlines were pressing, but what I gained out-weights what I gave and I want to thank all my colleagues of the current and previous editorial board for all the cooperative and stimulating meetings.

Hopefully, this self-report inspires you, the reader, to become an active member of the German AI community in the near future!

Sincerely yours, Ute Schmid

\section{Forthcoming Special Issues}

\section{Advances in Autonomous Learning}

Autonomous Learning research aims at understanding how adaptive systems can efficiently learn from the interaction with the environment, especially by having an integrated approach to decision making and learning, allowing systems to decide by themselves on actions, representations, hyper-parameters and model structures for the purpose of efficient learning.

It addresses challenges such as how to autonomously learn representations for efficient model use, how to arrive at suitable cost functions from meta-objectives (generalizing inverse RL), how to autonomously choose model structures and hyper-parameters in possibly non-stationary environments, or how to design efficient actor-reward strategies which generalize across tasks.

Application scenarios which require these type of complex models span high-impact domains such as robotics, life-long learning, intelligent tutoring, or big data analytics. We invite contributions related to the following non exhaustive list of topics: $\mathrm{s}$

- autonomous learning of rich data representations,

- active learning in structured (e.g., hybrid, relational) interactive domains,

- learning models with autonomous complexity adaptation,

- transfer learning,

- structure learning,

- statistical relational learning,

- theoretical advances to measure model autonomy,
- applications and project reports in the field of autonomous learning.

The Künstliche Intelligenz Journal, which is published and indexed by Springer, supports the following lists of formats: Technical contributions, research projects, discussions, dissertation abstracts, conference reports and book reviews.

If you are interested in contributing to this special issue, please contact one of the guest editors:

\section{Prof. Barbara Hammer}

Universitt Bielefeld

D-33594 Bielefeld

\section{Prof. Marc Toussaint}

Universitt Stuttgart

D-70569 Stuttgart

\section{Companion Technologies}

At present, we observe a rapid growth in the development of increasingly complex intelligent systems that serve users throughout all areas of their daily life. They range from classical technical systems such as household devices, cars, or consumer electronics through mobile apps and services to advanced service robots in various fields of application. While many of the rather conventional systems already provide multiple modalities to interact with, the most advanced are even equipped with cognitive abilities such as perception, cognition, and reasoning. However, the use of such complex technical systems and in particular the actual exploitation of their rich functionality remain challenging and quite often lead to users cognitive overload and frustration.

Companion Technologies aim at bridging the gap between the extensive functionality of technical systems and human users individual requirements and needs. They enable the construction of really smart adaptive, flexible, and cooperative technical systems by employing a combination of $\mathrm{AI}$ techniques and relying on psychological and neurobiological findings.

The special issue Companion Technologies of the KI Journal aims to present ongoing research, application perspectives, and other insights into an exciting research area emerging across the fields of Artificial Intelligence, Cognitive Psychology, and Cognitive Sciences. Topics of interest include, but are not limited to:

- Computational models of cognitive processes

- Reasoning for adaptive systems

- User-centered planning

- Multi-modal emotion and motivation recognition 
- Knowledge-based human computer interaction

- Knowledge-based dialogue management

- Cooperative and adaptive systems

The KI Journal, published and indexed by Springer, supports a variety of formats including technical articles, project descriptions, surveys, dissertation abstracts, conference reports, and book reviews. Interested authors are asked to contact the guest editors at their earliest convenience:

\section{Prof. Dr. Susanne Biundo-Stephan}

Daniel Höller

Pascal Bercher

Institute of Artificial Intelligence

Ulm University

89069 Ulm

susanne.biundo@uni-ulm.de

daniel.hoeller@uni-ulm.de

pascal.bercher@uni-ulm.de

\section{Semantic Web}

With more and more data available on the web, the use of semantic technologies is the key to making this knowledge accessible to machines. Thirteen years after the last special issue on the Semantic Web, it is time to review again the advances and state-of-the-art in this area. Several new standards for specifying data and schema information in a machine-processable way have emerged: the basic language to describe data, the resource description framework (RDF) with its schema extension RDFS has been revised in 2014; SPARQL became a widely used query language (SPARQL 1.1 standardised in 2013) and now also supports updates, retrieval of entailed query answers, and federation; the Web Ontology Language OWL has been revised and extended to OWL 2; and the rule interchange format RIF (standardized in 2013) allows for expressing rules in a common format. The recent Linked Open Data movement is based on these standards and makes a vast amount of interlinked resources available in the web and for use in semantically enriched applications.

What was still a far-away future when the first Semantic Web special issue appeared became reality: the big search engines now use semantic markup (via RDFa, microdata, or microformats) to improve their search results. With their joint schema.org initiative Google, Microsoft, and Yahoo! provide a unified schema for the structured description of web page content.

Topics of interest include, but are not limited to:

- Management of semantics and data on the Web, including Linked Data

- Languages, tools, and methodologies for representing and managing semantics and data on the Web

- Database, Information Retrieval, Information Extraction, Natural Language Processing and Artificial Intelligence techniques for the Semantic Web

- Searching and querying the Semantic Web

- Knowledge representation and reasoning on the Web

- Cleaning, quality assurance, and provenance of Semantic Web data, services, and processes

- Semantic Web data analysis

- Ontology-based data access and integration/exchange on the Web

- User Interfaces and interaction with semantics and data on the Web

- Information visualization of Semantic Web data

- Ontology engineering and ontology patterns for the Web

- Ontology modularity, mapping, merging, and alignment for the Web

- Trust, privacy, and security on the Semantic Web

The KI Journal, published and indexed by Springer, supports a variety of formats including technical articles, project descriptions, surveys, dissertation abstracts, conference reports, and book reviews.

Interested authors are asked to contact the guest editors at their earliest convenience:

Juniorprof. Dr. Birte Glimm

Institute of Artificial Intelligence

University of Ulm

89069 Ulm

birte.glimm@uni-ulm.de

\section{Prof. Dr. Heiner Stuckenschmidt}

Data- and Web Science Research Group

University of Mannheim

68159 Mannheim

heiner@informatik.uni-mannheim.de 